\title{
Crystal Structure of the High Temperature Phase of Strontium Barium Niobate
}

\author{
R. PAszkowski ${ }^{a *}$, M. Zubko ${ }^{a}$, K. Wokulska ${ }^{a}$, J. Kusz ${ }^{b}$ And J. DeC ${ }^{a}$ \\ ${ }^{a}$ Institute of Materials Science, University of Silesia, 75 Pułku Piechoty 1A, 41-500 Chorzów, Poland \\ ${ }^{b}$ Institute of Physics, University of Silesia, Uniwersytecka 4, 40-007 Katowice, Poland
}

\begin{abstract}
Pure and undoped strontium-barium niobate $\mathrm{Sr}_{0.40} \mathrm{Ba}_{0.60} \mathrm{Nb}_{2} \mathrm{O}_{6}$ ( $\mathrm{SBN} 40$ ) single crystals grown by the Czochralski method were investigated by single crystal X-ray diffraction methods. The study below $T_{\mathrm{C}}(429 \mathrm{~K}$ for SBN40) confirmed the structure with $P 4 b m$ space group. Above this temperature the structure transforms into the paraelectric, centrosymmetric one with $P 4 / \mathrm{mbm}$ space group. Analysis of the recorded diffraction patterns allowed to observe several signs of crystal structure modulation. On the registered diffraction images satellite reflections were found. A modulation vector $q=(\delta, \pm \delta$, ), where $\delta=0.3075(6)$ (at room temperature) was found and it was similar to that occurring in the SBN61. In addition, above the phase transition temperature on the $(h k)$ planes with $l$ integer a weak diffuse scattering was observed.
\end{abstract}

DOI: 10.12693/APhysPolA.130.856

PACS/topics: 42.70.Mp, 77.84.Dy, 61.50.-f, 71.55.Jv

\section{Introduction}

Strontium barium niobate $\mathrm{Sr}_{x} \mathrm{Ba}_{1-x} \mathrm{Nb}_{2} \mathrm{O}_{6}(\mathrm{SBN})$ is a multifunctional material which belongs to tetragonal tungsten bronzes family. Unique potential applications of SBN are due to its advantageous dielectric and electrooptic properties and a high photorefractive coefficient. This makes it a very interesting material for applications in optoelectronic and pyroelectric detectors, surface acoustic wave devices, or holographic data storage.

SBN crystallizes in a wide solid solution range $(0.26<$ $x<0.87$ ) [1] in the tetragonal symmetry (space group $P 4 b m)$. In ferroelectric phase, SBN has the $4 m m$ point group symmetry, which transforms to centrosymmetric $4 / m m m$ point group above $T_{\mathrm{C}}$. They also exhibit gradual crossover from conventional ferroelectric (SBN35) into utmost relaxor (SBN72) behavior [2]. So far, using the four-circle diffractometry the single crystals with a following compositions: SBN34, SBN47, SBN61, SBN82 [3], SBN50 [4] and SBN75 [5, 6] were investigated.

The aim of our studies was refinement of ferroelectric and paraelectric phase strontium-barium niobate $\mathrm{Sr}_{0.40} \mathrm{Ba}_{0.60} \mathrm{Nb}_{2} \mathrm{O}_{6}$ (SBN40) single crystals and specify the reasons for incommensurate modulation of crystal structure.

\section{Experimental}

As a subject of our studies we used samples prepared from a high quality single crystal of SBN40 $\left(\mathrm{Sr}_{x} \mathrm{Ba}_{1-x} \mathrm{Nb}_{2} \mathrm{O}_{6}\right.$, where $\left.x=0.40\right)$ obtained by the Czochralski method [2]. The samples were made from plates used earlier in precise lattice parameter measurements. Details of measuring principles were presented earlier $[7,8]$.

\footnotetext{
* corresponding author; e-mail: robert.paszkowski@us.edu.pl
}

After the lattice parameters measurements they were crushed in an agate crucible. Prepared in this way particles were attached to the glass capillary using hightemperature silicone. The X-ray measurement data were collected using a single crystal Xcalibur Kappa diffractometer (Oxford Diffraction) with a Sapphire3 CCD detector and a graphite-monochromatized Mo $K_{\alpha}(\lambda=$ $0.7107 \AA$ ) radiation. The measurements were carried out using a heating equipment (HotJet) in the temperature range from $330 \mathrm{~K}$ to $670 \mathrm{~K}$ in two cycles during heating and cooling. The structure refinement was carried out using full matrix least-squares method implemented in SHELXL software [9].

For precise study of the lattice parameters the single crystals have been earlier investigated by Bond method with the high accuracy and precision [7, 8]. Accurate temperature measurements of the lattice parameters allowed to determine the phase transition temperature [10]. The temperature was confirmed in measurements of electric susceptibility as a function of temperature and frequency [2].

\section{Results and discussion}

Basing on the obtained measurement data it was confirmed in accordance with the literature [3-6] that at room temperature SBN40 single crystals have the structure of space group P4bm. Carrying out the temperature measurements, it was found that the above phase transition temperature (for SBN40 it is $429 \mathrm{~K}$ [9]) the structure transformed into the paraelectric, centrosymmetric symmetry with $P 4 / \mathrm{mbm}$ space group, which is also in accordance with previous works [3-5]. Results of refinement of the crystal structures were shown in Table I.

Analysis of recorded diffraction patterns allowed to observe several signs of crystal structure modulation described earlier by Bursill and Lin [11]. First, it was noted that close to the major reflections on planes $\{h, k, l \pm\}$ 
TABLE I

Results of refinement of crystal structure of SBN40 single crystals.

\begin{tabular}{|c|c|c|}
\hline SBN40 single crystals & $300 \mathrm{~K}$ & $600 \mathrm{~K}$ \\
\hline crystal system & tetragonal & tetragonal \\
\hline$a[\AA]$ & $12.4791(2)$ & $12.5072(2)$ \\
\hline$b[\AA]$ & $12.4791(2)$ & $12.5072(2)$ \\
\hline$c[\AA]$ & $3.9615(2)$ & $3.9541(2)$ \\
\hline$\alpha\left[^{\circ}\right]$ & 90 & 90 \\
\hline$\beta\left[^{\circ}\right]$ & 90 & 90 \\
\hline$\gamma\left[\left[^{\circ}\right]\right.$ & 90 & 90 \\
\hline unit cell volume $\left[\AA^{3}\right]$ & $616.92(4)$ & $618.54(4)$ \\
\hline space group & $P 4 b m$ & $P 4 / m b m$ \\
\hline Radiation type & $\operatorname{MoK} \alpha$ & $\operatorname{MoK} \alpha$ \\
\hline absorption coefficient $\mu\left[\mathrm{mm}^{-1}\right]$ & 33.214 & 14.668 \\
\hline No. of reflections measured & 6665 & 3484 \\
\hline No. of independent reflections & 1173 & 1084 \\
\hline$R_{\text {int }}$ & 0.0245 & 0.0289 \\
\hline final $R_{1}$ values $(I>2 \sigma(I))$ & 0.0426 & 0.0238 \\
\hline final $w R\left(F^{2}\right)$ values $(I>2 \sigma(I))$ & 0.1101 & 0.0600 \\
\hline final $R_{1}$ values (all data) & 0.0435 & 0.0249 \\
\hline final $w R\left(F^{2}\right)$ values (all data) & 0.1107 & 0.0605 \\
\hline
\end{tabular}

much weaker satellite reflections were found. To better illustrate phenomena the reconstructions of the reciprocal space for the three planes in two different temperatures of $300 \mathrm{~K}$ and $380 \mathrm{~K}$ were performed (Fig. 1).

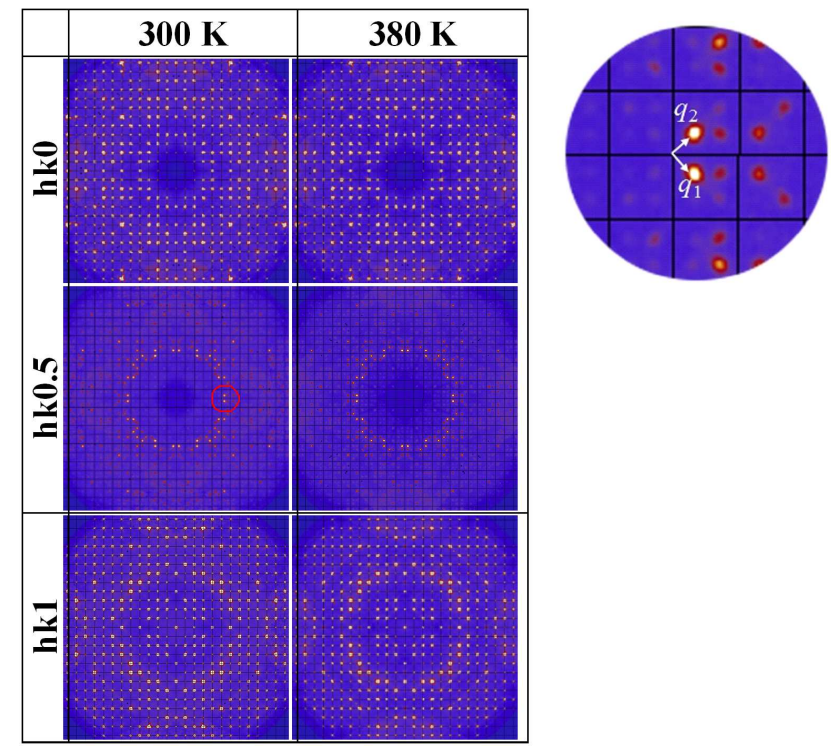

Fig. 1. At the left: a visualization of reciprocal space on planes $\{h, k, l \pm\}$. The picture besides shows the sector with the selected modulation vectors $q_{1,2}=$ $\left(\delta, \pm \delta, \frac{1}{2}\right)$, where $\delta=0.3075(6)$.

On $\{h, k, l \pm\}$ planes, satellite reflections were found. A modulation vector $q=(\delta, \pm \delta$, $)$, where $\delta=0.3075(6)$ (in room temperature) was found. It was similar to that occurring in the SBN61 which corresponds to the earlier work [12]. Further analysis of the intensity changes in the three areas (near satellite reflection $\left(5 \frac{1}{3}, \overline{\frac{1}{3}}, \overline{\frac{1}{2}}\right)$, the main reflection $(6,2, \overline{1})$ and a background) was carried out in the temperature range of 330-670 K (Fig. 2).

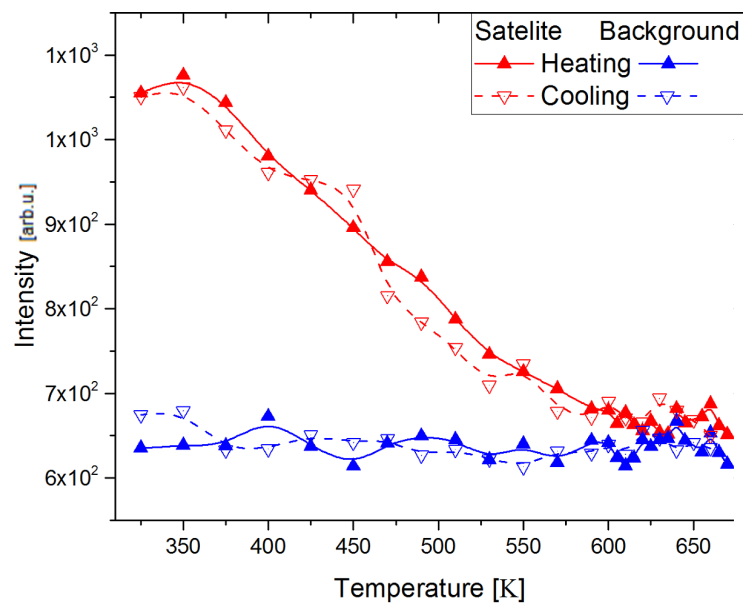

Fig. 2. Intensity changes in the two areas in the temperature range $330-670 \mathrm{~K}$. The red lines describes the changes of the satellite reflection while the blue lines describes changes of the background. The full triangles determined measurement data collected during the heating. The open triangles showed measuring data during cooling.

The measurements were performed for two cycles during heating and cooling. Basing on the intensity changes in a function of temperature, it was found that the intensity of all reflections decreases with increase of temperature. The intensity of satellite reflection in the vicinity of $610 \mathrm{~K}$ was equal with the background.

Occurring phenomenon, "blanking" of satellite reflections may be tantamount to the disappearance of incommensurate modulated structure of SBN single crystals. A similar phenomenon was observed in SBN70 by Balagurov et al. [13] where satellite reflections disappeared at $773 \mathrm{~K}$. Lowering the temperature to the room temperature resulted in the appearing of the satellite reflections. Basing on the obtained results it was shown that the temperature at which the satellite reflections "disappear" depends on the crystal stiochiometry and it has different values. Disappearance of the signs of incommensurate modulation can be also explained by a smaller amount of nanoregions, whose number according to the literature [14] decreases with increase of the strontium content in the pentagonal tunnel of crystal structure.

Additionally, rather weak diffuse scattering was observed which is a sign of a disorder of periodicity of the crystal lattice. In the case of strontium barium niobate, this phenomenon can be observed only in the measurement above the phase transition temperature. Discovered diffuse scattering was observed on the $(h k)$ planes with $l$ integer. Measurement frames recorded at room 
temperature and above the phase transition, where signs of diffuse scattering were observed, are shown in Fig. 3.

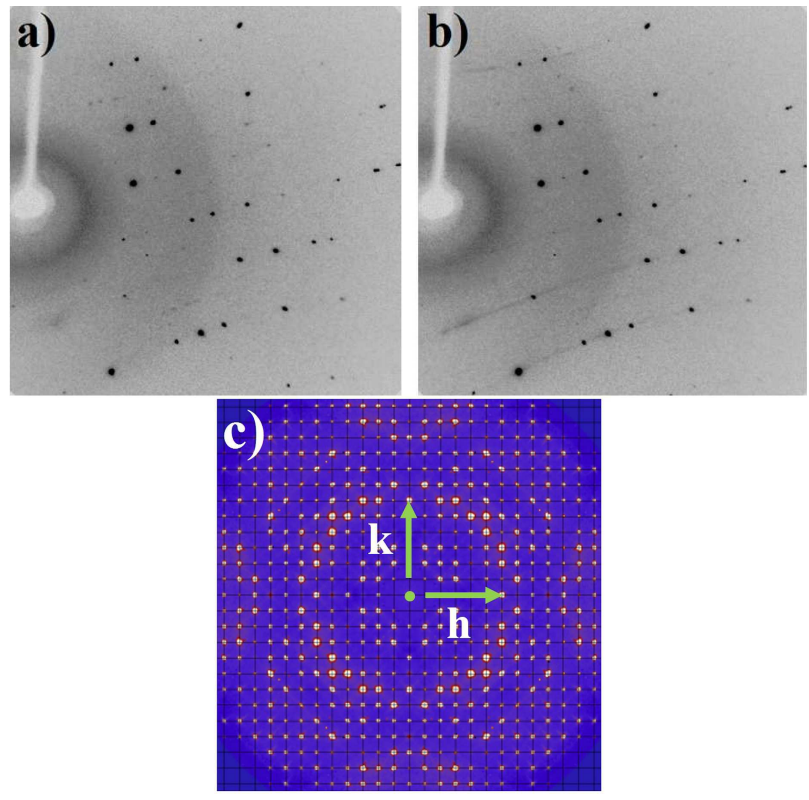

Fig. 3. Measurement frames at room temperature $300 \mathrm{~K}(\mathrm{a})$ and above the phase transition - $500 \mathrm{~K}(\mathrm{~b})$. Part (c) shows a reconstruction of the reciprocal space for the $h k l$ plane above the phase transition temperature.

Reconstruction of the reciprocal space presented in Fig. 3c shows decrease of diffuse scattering intensity from the centre of the image (marked in Fig. 3c as green dot). That displacive disorder behavior can be related to the reorganization of the oxygen octahedrons. These results were confirmed for SBN61 using neutron diffraction technique [12]. However, these features are not visible strongly enough to exclude an occupational disorder. The results of measurements obtained by Schefer et al. [15] shown that the amplitude of occupational modulation of the $\mathrm{NbO}_{6}$ octahedrons decrease with increase of the barium concentration in the crystal structure. This indicates that the reason of modulation is the ability to occupy pentagonal tunnels by strontium and barium atoms. It was confirmed by Viehland et al. [16], who used the transmission electron microscope. Observed by the authors incommensurate reflection explained the occurrence of ferroelectric nanodomains, which may be the reason of existence of the described phenomena.

Presented in this article results are preliminary and require further investigation.

\section{Conclusions}

The study at room temperature confirmed that the SBN40 single crystals have the structure with space group $P 4 b m$. Above temperature $T_{\mathrm{C}}(429 \mathrm{~K}$ for SBN40) the structure transforms into the paraelectric, centrosymmetric one with space group $P 4 / \mathrm{mbm}$. Basing on recorded diffraction patterns, several signs of crystal structure modulation were found. Determined temperature at which the satellite reflections "disappear" was not equal to results obtained on other crystals with different stoichiometry. Earlier signs of disappearance of incommensurate modulation can be explained by a smaller amount of nanoregions. Basing on analysis of the diffuse scattering, it was found that the described phenomena may be related to displacive disorder associated with reorganization of the oxygen octahedrons and also occupational modulation associated with ability to occupancy pentagonal tunnels by strontium and barium atoms.

\section{References}

[1] M. Ulex, R. Pankrath, K. Betzler, J. Cryst. Growth 271, 128 (2004).

[2] T. Łukasiewicz, M.A. Świrkowicz, J. Dec, W. Hofman, W. Szyrski, J. Cryst. Growth 310, 1464 (2008).

[3] S. Podlozhenov, H.A. Graetsch, J. Schneider, M. Ulex, M. Wöhlecke, K. Betzler, Acta Crystallogr. B 62, 960 (2006).

[4] T.S. Chernaya, T.R. Volk, I.A. Verin, L.I. Ivleva, V.I. Simonov, Crystallogr. Rep. 47, 213 (2002).

[5] P.B. Jamieson, S.C. Abrahams, J.L. Bernstein, J. Chem. Phys. 48, 5048 (1968).

[6] T.S. Chernaya, B.A. Maksimov, T.R. Volk, L.I. Ivleva, V.I. Simonov, Phys. Solid State 42, 1716 (2000).

[7] R. Paszkowski, K. Wokulska, J. Dec, T. Łukasiewicz, J. Cryst. Growth 401, 327 (2014).

[8] R. Paszkowski, K. Wokulska, J. Dec, Solid State Phenom. 203-204, 77 (2013).

[9] G.M. Sheldrick, Acta Crystallogr. A 64, 112 (2008).

[10] R. Paszkowski, K. Wokulska, T. Łukasiewicz, J. Dec, Cryst. Res. Technol. 48, 413 (2013).

[11] L.A. Bursill, P.J. Lin, Acta Crystallogr. B 43, 49 (1987).

[12] Th. Woike, V. Petricek, M. Dusek, N.K. Hansen, P. Fertey, C. Lecomte, G. Chapuis, M. Imlau, R. Pankrath, Acta Crystallogr. B 59, 28 (2003).

[13] A.M. Balagurov, F. Prokert, B.N. Savenko, Phys. Status Solidi A 103, 131 (1987).

[14] V.V. Shvartsman, J. Dec, S. Miga, T. Łukasiewicz, W. Kleemann, Ferroelectrics 376, 1 (2008).

[15] J. Schefer, J.D. Schaniel, V. Petricek, Th. Woike, A. Cousson, M. Woehlecke, Z. Kristallogr. 223, 399 (2008).

[16] D. Viehland, Z. Xu, W.-H. Huang, Philos. Mag. A 71, 205 (1995). 